\title{
Recognition memory for nonobject drawings
}

\author{
LIEN-CHONG MOU and NANCY S. ANDERSON \\ University of Maryland, College Park, Maryland \\ W. S. VAUGHAN, JR. \\ Office of Naval Research, Arlington, Virginia \\ and \\ RICHARD O. ROUSE, JR. \\ Williams College, Williamstown, Massachusetts
}

\begin{abstract}
Following a procedure similar to Shepard's (1967), 39 subjects studied 80 nonobject drawings for 2 or 6 sec each. The drawings had been previously rated for object-like appearance following the procedure by Kroll and Potter (1984). Recognition of the studied items was tested by showing 80 drawings one at a time: 40 selected from the 80 in the study set and 40 new drawings. Both sets had an equal number of drawings rated low, medium, or high in object likeness. The subjects responded "old" or "new" and gave confidence ratings immediately and $24 \mathrm{~h}$ later. A subset of 18 old drawings from the study set was selected to be named by subjects following the delayed test. Recognition data showed that memory was better for the drawings that had been shown at the longer study duration and were more object-like. A measure of name agreement was found to be correlated with object-like appearance, showing higher name agreement for more objectlike drawings. Relationships among object likeness, name agreement, and memorability of nonobject drawings are discussed.
\end{abstract}

Studies of memory for visual pictures, objects, and other complex meaningful forms have shown that meaningful, familiar pictures or visual information can be recognized after a single presentation and can be discriminated from pictures not previously seen with a high degree of accuracy (96.7\%) (Shepard, 1967). In addition, Nickerson (1965) found that subjects who were shown an extended sequence of photographs of various contents were able to identify those that occurred for a second time within the sequences with $87 \%$ accuracy, even with a maximum lag between items of 200 photographs. Thus, it appears that the capacity for visual information in recognition memory is very high, relative to that for verbalized units. However, it is not clear just what are the important properties of these stimuli, in addition to meaningfulness, that allow such high levels of recognition performance.

Kroll and Potter (1984) developed a set of nonobject drawings for use in a decision task to compare lexical, object, and reality decisions. They found that both lexicaland object-decision tasks produced similar response latencies. In addition, these nonobject forms were rated by the subjects according to their "looking like a real object." The experimenters found a significant negative correlation between rejection time and rated-object similarity,

The authors wish to thank Alva Hughes for her help in drawing the nonobject stimuli, and Cara Forrest, Lisa Hamel, and Chris Bresnahan for collecting and scoring the data. Computer time was provided in full by the Computer Science Center. Address correspondence to Nancy S. Anderson, Department of Psychology, University of Maryland, College Park, MD 20742. which indicated that nonobjects that resembled real objects were difficult to reject as distractors in the lexicaldecision task.

The present experiment was conducted to investigate the recognition memory of these nonobject drawings using a yes/no recognition procedure.

\section{METHOD}

\section{Subjects}

Thirty-nine college students volunteered to participate and received extra course credits. There were 22 subjects in the 2 -sec group and 17 in the 6-sec group.

\section{Materials and Procedure}

The nonobject drawings were generated by arbitrarily combining different segments of real-object line drawings taken from Snodgrass and Vanderwart (1980). A total of 280 nonobject drawings, including 88 from Kroll and Potter (1984), were shown on pages of a booklet to 54 subjects who rated them on a 7-point scale. The scale descriptions indicated that each drawing was to be rated " 1 " if it looked very much like a real object and " 7 " if it looked nothing like a real object. The average ratings of the drawings were categorized into three groups: high, medium, and low object-like drawings. A study set and a comparable distractor set of $\mathbf{8 0}$ drawings, each of which consisted of 27 randomly chosen patterns from each of the high and low object-like categories and 26 randomly chosen patterns from the medium object-like category, were used in the memory study. All drawings were prepared on slides and presented to the subjects by a projector on a rear-projection screen. The subjects were tested in groups of 1,2 , or 3 , and were randomly assigned to one of two rates of presentation of the study set: $2 \mathrm{sec}$ or $6 \mathrm{sec}$ per slide.

The subjects were asked to study and remember each of the 80 drawings for a recognition test that would follow the single-presentation study set. Two recognition tests were used. One test was given immediately after the study set and consisted of $\mathbf{8 0}$ drawings, $\mathbf{4 0}$ from the study set 
and $\mathbf{4 0}$ from the distractor set. For this immediate test, the $\mathbf{4 0}$ study or distractor drawings consisted of 14,13 , and 13 drawings from the low, medium, and high object-like drawings, respectively. The other recognition test, consisting of the other $\mathbf{4 0}$ drawings from the study set and another 40 different distractor drawings, was given $24 \mathrm{~h}$ after the study set. For this delayed test, the distribution of drawings was 13, 13 , and 14 from the low, medium, and high categories, respectively.

In both recognition tests, the subject was to respond to each 6-sec presentation of a drawing with "old" if it had been in the study set or "new" if it had not been in the study set. Responses were recorded on a sheet of paper, and the subjects also indicated their confidence in their responses on a 3-point scale.

After the second recognition test, a booklet containing 18 drawings from the study set (9 each of low and high object-like drawings) was given to each subject. The subjects were asked to name the objects that each drawing reminded him/her of most, and to indicate the kinds of parts or features that occurred in each drawing. An imagery scale (VVIQ) was then given to each subject to rate his/her own imagery ability with eyes open and eyes closed.

\section{RESULTS}

The proportion of errors, the hit rate, and the falsepositive rate for individual subjects was calculated for each set of the high, medium, and low object-like categories in the immediate recognition test and the 24-h delay recognition test. The average proportion of errors and the average hit rate for each of the experimental conditions of time presentaton are shown in Figure 1.

A $2 \times 2 \times 3$ (presentation rate $\times$ delay $\times$ object likeness) analysis of variance for errors and hit rate was conducted. The main effect of rate of presentation was significant for proportion of errors, and showed that errors were higher for the shorter presentation of $2 \sec (.30)$ than for the longer presentation of $6 \sec (.18)[F(1,37)=$ 24.31, $\left.p<.0001, M S_{\mathrm{e}}=.03\right]$. The main effect of delay indicated fewer errors on the immediate test (.21) than on the delayed recognition test $(.29)[F(1,37)=35.85$, $\left.p<.0001, M S_{\mathrm{e}}=.008\right]$. Finally, the main effect of object likeness was also significant; fewer errors were made for items in categories with ratings that were more objectlike (high $=.21$, medium $=.24$, low $=.30)[F(2,74)$ $\left.=40.87, p<.0001, M S_{\mathrm{e}}=.004\right]$. However, the interaction of delay and object likeness was found to be significant, showing smaller increases of errors by delay for the medium object-like category (.03) than for the high (.13) or low $(.06)$ object-like categories $[F(2,74)=5.13$, $p<.01, M S_{\mathrm{e}}=.008$ ].

The analysis of the hit rate showed the same main effects and interaction effect as those for errors. The data on confidence ratings were combined with the hit rate and false-positive rate to give $A_{g}$, a sensitivity measure for memory operating characteristic, and this measure yielded results consistent with those for errors and hit rate.

For the 18 drawings named by the subjects, an uncertainty measure (Snodgrass \& Vanderwart, 1980) was used to indicate the degree of name agreement for each drawing. The uncertainty measure, $H$, was based on the number of different names and the proportion of subjects who gave each name for each drawing. A high $H$ value indicates more different names given and less name agreement, whereas a low $H$ value reflects better name agree-

\section{Proportion of errors as a function of object likeness and delay conditions}

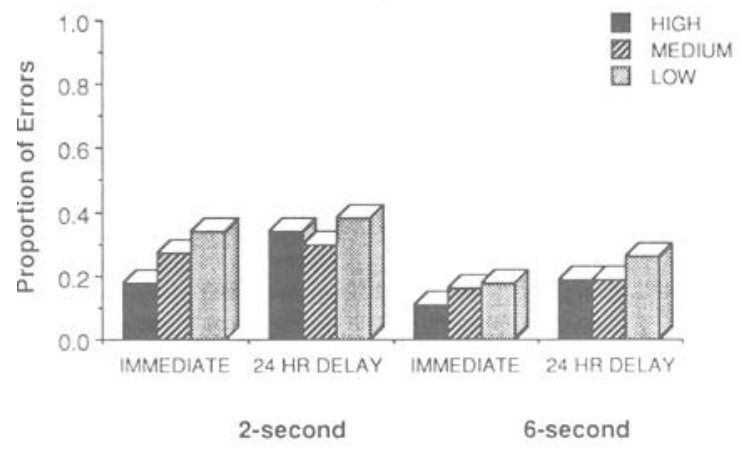

Hit rate as a function of object likeness and delay conditions

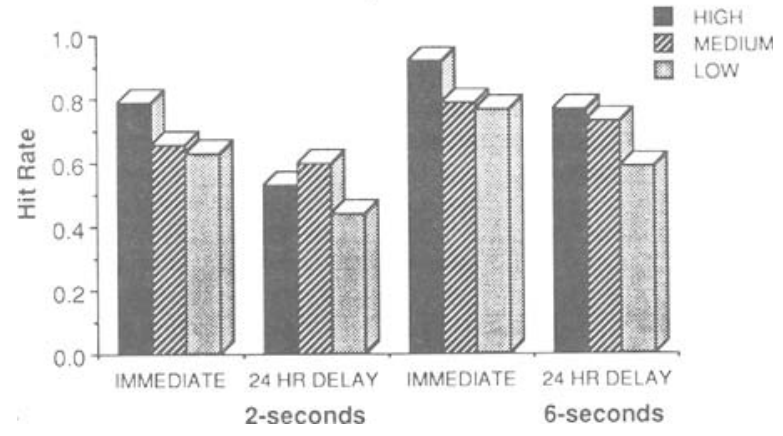

Figure 1. Proportion of errors and hit rate as a function of object likeness and delay conditions.

ment. Three judges categorized the names and calculated the $H$ for each drawing based on responses from both the 2 -sec and the 6-sec groups. The interrater agreement on $H$ ranged from .95 to .99 . The average $H$ for each drawing was then used to determine if name agreement was correlated with object-like rating. The correlation between name agreement $(H)$ and object-like rating was found to be $.82(p<.001)$, which indicates that the more objectlike drawings also had higher name agreement.

The correlations between the overall imagery scores on the VVIQ and correct recognitions for immediate and delayed testing showed no significant relationships for subjects who studied the drawings at the 6-sec rate. Only two significant correlations of +.44 for number correct on the immediate recognition test and "closed" ' imagery score, and number correct on the delayed recognition test and "open" imagery score, were found for the 2-sec group. An examination of the scatter diagram for these two positive correlations showed somewhat curvilinear relationships with linearity (low imagery and high correctness) apparently due primarily to a few low-imagery subjects.

\section{DISCUSSION}

The results indicated that the subjects recognized nonobject drawings with relatively high accuracies of $74 \%$ (immediate recognition test) and 
$66 \%$ (delayed recognition test) when the drawings were presented at a 2-sec rate for a single viewing. Recognition accuracies were $85 \%$ for the immediate test and $79 \%$ for the 24-h delay test when the drawings were presented at a 6-sec rate for a single viewing. These accuracies are lower than those found by Shepard (1967), who found $96.7 \%$ accuracy immediately after presentation and $92 \%$ after a 72 -h delay. Furthermore, we predicted that the drawings rated more object-like were, on the average, more likely to be recognized (79\%) and that those drawings rated lower in object likeness were less likely to be recognized (72\%). Clearly, these line drawings did not provide "visual richness" (Anglin \& Levie, 1985) and thus did not yield the perfect recognition that was found by Shepard for picture memory of color photographs viewed once for an average $5.9 \mathrm{sec}$.

The finding that the drawings previously rated more object-like were better recognized provides some support for the dual-coding formulation of Paivio (1971), inasmuch as the more object-like drawings provided both a visual and a verbal representation of the same drawing. The data showing a correlation of .82 between naming consistencies (given after the last recognition test of these nonobject drawings) measured by an uncertainty metric and prior object-like ratings indicate that the drawings rated more object-like had higher name agreement (these drawings were also more memorable). These naming data provide information about what details were encoded but not about when (e.g., study or test) they were encoded, as was investigated by Loftus and Kallman (1979). The uncertainty metric used here was a measure of global name agreement. More data on detail naming in the study or test phase would need to be collected to support encoding consistencies in either of these phases. Detailed encoding operations might explain the interaction found for delay and object likeness that indicated more correct responses for the medium object-like drawings during delayed recognition in the 2 sec condition.
The lack of consistent relationships between imagery scores as measured by the VVIQ and correct recognition may be due to individual differences, or may indicate that global measures of imagery vividness mask the relationship between imagery and memory, as discussed by Ernest (1983).

\section{REFERENCES}

ANGLIN, G. J., \& LeVIE, W. H. (1985). Role of visual richness in picture memory. Perceptual \& Motor Skills, 61, 1303-1306.

ERNEST, C. H. (1983). Imagery and verbal ability and recognition memory for pictures and words in males and females. Educational Psychology, 3, 227-244.

Kroll, J. F., \& PoTter, M. C. (1984). Recognizing words, pictures, and concepts: A comparison of lexical, object, and reality decisions. Journal of Verbal Learning \& Verbal Behavior, 23, 39-66.

LofTUS, G. R., \& KallmaN, H. J. (1979). Encoding and use of detail information in picture recognition. Journal of Experimental Psychology: Human Learning \& Memory, 5, 197-211.

NiCKERSON, R. S. (1965). Short term memory for complex meaningful visual configurations: A demonstration of capacity. Canadian Journal of Psychology, 2, 155-160.

Paivio, A. (1971). Imagery and verbal processes. New York: Holt, Rinehart \& Winston.

SHEPARD, R. (1967). Recognition memory for words, sentences and pictures. Journal of Verbal Learning \& Verbal Behavior, 6, 156-163.

SNOdGRAsS, J. G., \& VANDERWART, M. (1980). A standard set of 260 pictures: Norms for name agreement, familiarity, and visual complexity. Journal of Experimental Psychology: Human Learning \& Memory, 6, 174-215. 